\title{
The Formation of Massive Stars and Star Clusters
}

\author{
Cara Battersby ${ }^{* \dagger}$ \\ Harvard-Smithsonian Center for Astrophysics \\ E-mail: cbattersbyecfa.harvard.edu
}

The life cycle of stars and gas in the Milky Way illuminates and shapes our view of the universe. This cycle is driven largely by massive stars through their immense ionizing radiation, powerful winds and outflows, and explosive supernovae, yet the processes leading to their formation remain elusive. I review the status of our understanding of massive star and cluster formation, beginning with a theoretical framework outlining the varying modes proposed for the accumulation of material onto forming stars: core accretion and competitive accretion. The observable consequences of each theory and their current statuses are discussed. I then delve into the growing body of observations toward massive star and cluster forming regions, focusing on recent observations of the structure and evolution of cluster- forming regions at early stages. I conclude with an outlook for the next stages in the field of massive star formation.

Frank N. Bash Symposium 2013: New Horizons in Astronomy (BASH 2013)

October 6-8, 2013

Austin, Texas

* Speaker.
†SMA Postdoctoral Fellow. 


\section{Motivation and Overview}

Massive stars, while less plentiful than their low-mass counterparts, exert enormous influence over their surroundings. Through their immense ionizing radiation, powerful winds and outflows, and explosive supernovae, massive stars shape the universe around us. The light we see from other galaxies is dominated by the light from massive stars and much of what we understand about star formation over cosmic time hinges on our local conception of the formation massive stars. Moreover, massive stars are the precursors to black holes and the origin of most of the metals in the universe. Our understanding of their natal environment and formation mechanisms, while fundamental to many branches of astrophysics, remains rudimentary.

Our picture of massive star and cluster formation remains incomplete due to the observational difficulty and theoretical complexity of the problem. Massive stars form and grow while deeply embedded in dense clouds of gas and dust. Massive stars are inherently rare and evolve very quickly, meaning that one needs to look comparably further away and over a much larger area to get the same statistics as for low-mass stars. Confusion and extinction present a challenge while the star remains in its dense natal cocoon and the rapid destruction of this natal cocoon as the star forming region evolves presents another challenge. The observational hurdles are being overcome with the next generation of telescopes that allow us to observe massive star formation on large scales (Galactic Plane Surveys) and at high-resolution (sub-mm and radio interferometry). On the theoretical side, improved computing power and inclusion of more detailed physics (stellar feedback, full MHD 3-D simulations) is producing increasingly more realistic physical models of massive star and cluster formation.

I review here the current status of our understanding of massive star formation, in particular, I present some predominant theories on massive star formation and various observational tests to distinguish between them. None of these tests have proved conclusive but new models matched with improved observational capabilities should provide resolution in the next decade.

\section{Introduction to Massive Star Formation Theory}

The predominant (and perhaps only) mode of massive star formation is in clusters (Lada \& Lada, 2003; de Wit et al., 2005), and a definitive understanding of star cluster formation has been a long-sought goal in astrophysics. A global picture of star formation must include the process that turns Giant Molecular Clouds (GMCs) into young stellar clusters and associations. Fundamental questions concerning the formation mechanism, environment, and early evolution of stellar clusters have remained unanswered due to the inherent complexity of the problem. Forming star clusters are embedded $\left(\mathrm{A}_{V} \sim 10-100\right)$, short-lived, and often distant $(\sim 5 \mathrm{kpc})$. The problem is hierarchical, and by its very nature spans many physical scales, from parsec-scale molecular clumps spread throughout the Galaxy, down to cores on $<0.1 \mathrm{pc}$ scales.

The formation of low-mass stars has been explored in detail in nearby low-mass star-forming regions and is understood to proceed through some well-constrained phases (see Figure 1 and Shu et al., 1987). Cores condense within molecular clouds and lose magnetic and turbulent support through ambipolar diffusion. A protostar forms in the center of the collapsing core, which is surrounded by a nebular accretion disk. The angular momentum loss in this disk is counteracted 


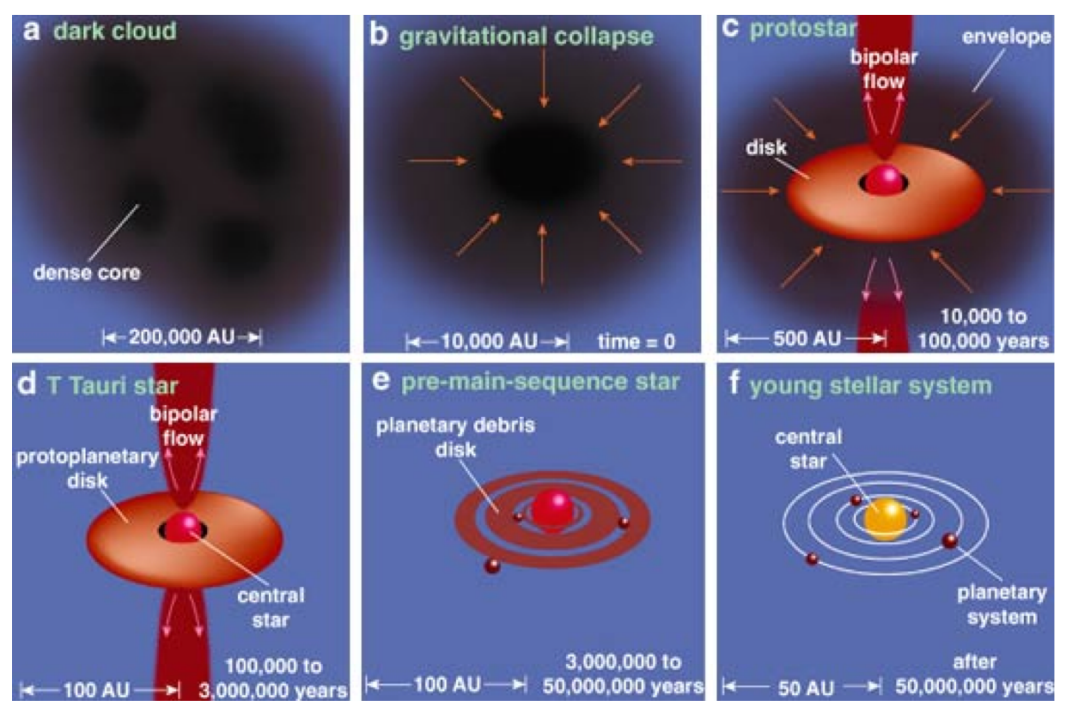

Figure 1: The basic model of isolated low mass star formation, based on the theory developed by Shu et al. (1987). This model cannot simply be scaled up to explain massive star $\left(M>8 M_{\odot}\right)$ formation as a spherically symmetric collapsing core quickly exceeds the Eddington limit and prevents further collapse. Krumholz et al. (2009) showed that this problem can be solved by invoking 3-D instabilities which allow radiation to escape in bubbles while the star continues to accrete along dense filaments. One current theory of massive star formation, core accretion, suggests that massive star formation is a scaled up version of this picture in which the massive core is supported by non-thermal (turbulent and magnetic) pressure. Cartoon from T. Green (American Scientist, Jul-Aug 2001).

by the formation of bipolar outflows emanating from the protostar poles. As the protostar turns on, heats up, and evaporates the remaining core envelope, the system reveals a newly formed star surrounded by a circumstellar disk.

While the paradigm of low-mass star formation is generally accepted (see Figure 1), the formation mechanism of massive stars remains a topic of debate. A simplistic, spherically symmetric, scaled-up massive core cannot collapse to form a massive star $\left(M>8 \mathrm{M}_{\odot}\right)$ akin to low-mass star formation, as the energy released through gravitational contraction quickly exceeds the Eddington luminosity and prevents further collapse (e.g. Shu et al., 1987). Krumholz et al. (2009) suggested that this problem can be circumvented by allowing material to flow onto the massive core through dense filaments, while energy escapes in radiatively driven bubbles.

While some argue that the formation of massive stars may be a scaled-up version of low mass star formation, with a hydrostatic massive core supported by turbulent pressure (the turbulent core accretion model; McKee \& Tan, 2003; McLaughlin \& Pudritz, 1996), others argue that the formation mechanism of stars in clusters is a unique and competitive process (competitive accretion; Bonnell et al., 2001; Wang et al., 2010). The turbulent core accretion model of McKee \& Tan (2003) suggests that massive stars form from massive hydrostatic cores supported by the non-thermal pressure provided by turbulence and magnetic fields. This high level of non-thermal pressure support naturally arises in massive, gravitationally bound gas clumps ${ }^{1}$, many of which may form clusters of

\footnotetext{
${ }^{1}$ In this work, we use the terminology wherein clouds are roughly $10^{3}-10^{4} \mathrm{M}_{\odot}$ and 2-15 pc, clumps are roughly $10^{2}-10^{3} \mathrm{M}_{\odot}$ and 0.3-3 pc, and cores are roughly 1-100 $\mathrm{M}_{\odot}$ and 0.03-0.2 pc as in Bergin \& Tafalla (2007).
} 


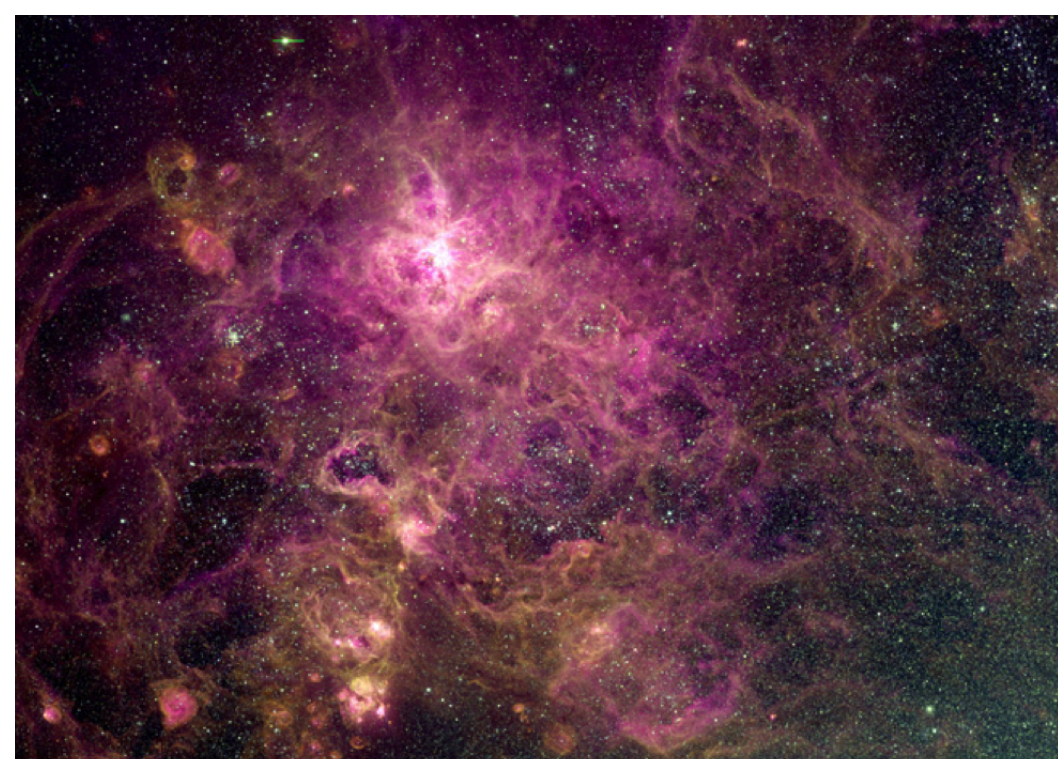

Figure 2: An image of 30 Doradus exemplifying the complex environment of massive star formation. This image of 30 Doradus, the Tarantula Nebula, in the Large Magellanic Cloud (LMC) was taken with the Curtis Schmidt telescope at Cerro Tololo Inter-American Observatory (CTIO) in Chile, as part of the Magellanic Cloud Emission Line Survey (MCELS) project. This color image was produced using three separate exposures taken in hydrogen (red), sulfur (green), and oxygen (blue) filters. Image credit: S. Points, C. Smith, R. Leiton, C. Aguilera and NOAO/AURA/NSF

stars. The theory of competitive accretion, developed from the simulations of Bonnell et al. (2001), suggests instead that massive stars form from small sub-virial seeds that compete for material in a massive gas clump. Those seeds at advantageous locations within the clump will accrete more material, forming massive stars.

Differentiating between competing theories of massive star formation has been hampered by the inherent complexity of the problem (see, e.g., Figure 2). Star formation is hierarchical and spans many orders of magnitude in size scale $(<0.1 \mathrm{pc}$ scale cores enveloped within $1 \mathrm{pc}$ scale clumps distributed on kpc scales throughout the Galaxy), density $\left(\mathrm{n}_{\text {clump }} \sim 10^{3} \mathrm{~cm}^{-3}-\mathrm{n}_{\text {core }} \sim\right.$ $\left.10^{7} \mathrm{~cm}^{-3}\right)$, and mass $\left(\sim 10 \mathrm{M}_{\odot}\right.$ cores to $10^{4} \mathrm{M}_{\odot}$ clumps). Simulations are also complicated by the varied physical properties important in their formation, including, but not limited to, stellar feedback, turbulence, gravity, radiative transfer, and magnetohydrodynamics. Observationally, the problem is complicated by the embedded nature and relative rarity of massive star-forming regions, necessitating large samples, high resolution long-wavelength observations toward distant, embedded sources. Through a combination of only forming a small fraction of massive stars in a standard initial mass function and the short lifetimes of massive stars, massive star-forming regions are relatively rare, meaning that they are further away on average and require high resolution facilities. The short-lived evolutionary phases also require large samples to determine an evolutionary sequence and timescale. The earliest phases of massive star formation are highly embedded ( $\left.A_{V} \sim 10-100\right)$, necessitating long-wavelength observations of re-processed light from dust grains. Furthermore, once a massive star forms, it evolves rapidly, quickly heating, ionizing, and eventually destroying the natal environment from which it formed. 

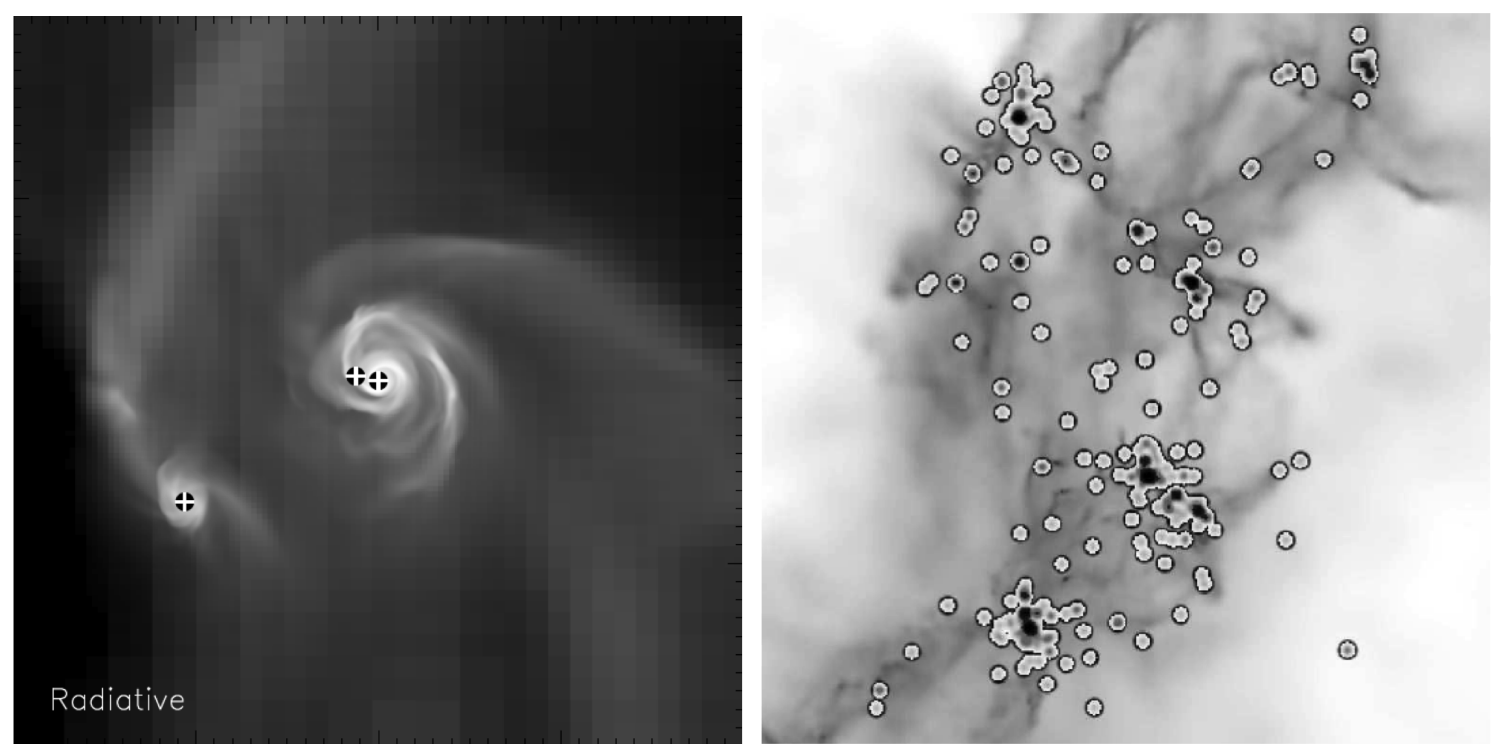

Figure 3: Core Accretion (Left) vs. Competitive Accretion (Right). The image on the left shows the formation of an individual massive stellar system (protostars marked with a + sign) within a massive bound core (0.02 pc box) in this simulation from Krumholz et al. (2007). The image on the right shows a cluster forming clump ( 1 pc box) and the large reservoirs out of which stars can accrete material. The most massive stars are formed preferentially in regions favorable to accreting a large amount of mass and are not confined to bound cores (from Bonnell et al., 2007, 2003).

\subsection{Core Accretion}

The core accretion model for massive star formation (e.g.; McKee \& Tan, 2003; McLaughlin \& Pudritz, 1996; Krumholz et al., 2007) is similar to our model for low-mass star formation, but scaled up in mass. This increase in mass (and therefore gravitational energy pushing for collapse) is counteracted by an increase in pressure working against collapse. These massive, hydrostatic cores are supported by the non-thermal pressure provided by turbulence and magnetic fields and will each collapse to form a single star or bound stellar system. In this model, the collapse of hydrostatic, bound cores is a slow, equilibrium process.

In this top-down fragmentation from molecular clouds to clumps to cores to stars, the cores represent the smallest bound structures, each one forming a single star or bound stellar system (see Figure 3). Since stars form directly from their natal cores in this model, the core mass function should map directly onto the stellar initial mass function, modulo some efficiency factor. Similarly, the spatial and velocity distribution of young stars should closely match that of cores. Finally, the overall star formation efficiency within a cloud or Galaxy is expected to be low since star formation is only occurring in cores (e.g.; Krumholz \& Bonnell, 2007).

One main distinguishing feature of the core accretion model is that the reservoir out of which all stars form is confined to their surrounding cores. The formation of a massive star requires the existence of a massive core. A large amount of non-thermal (turbulent or magnetic) pressure is required to stabilize such a core. In the core accretion model, such a core may form in isolation, meaning that the formation of massive stars in isolation, while unlikely, is possible. 


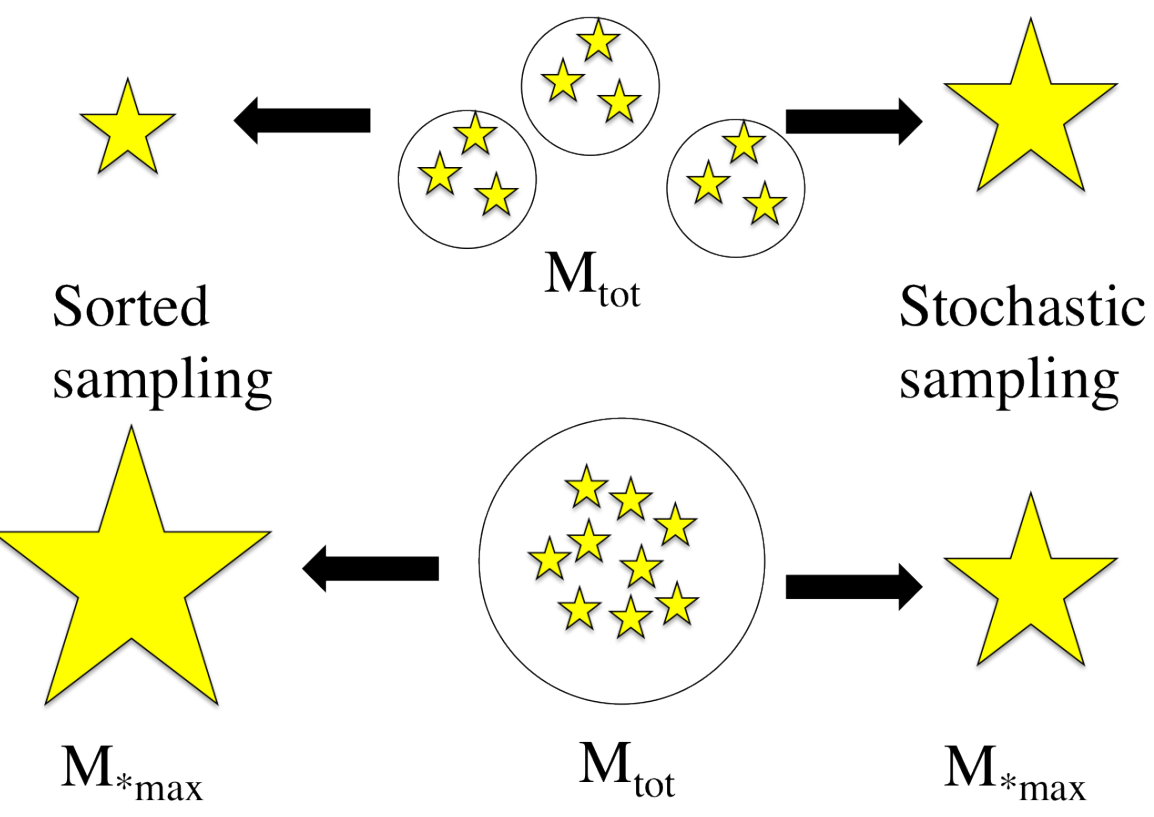

Figure 4: In the top panel, three star clusters form with a total mass (both gas and eventually stars) equaling that of a single massive star cluster, below. In the sorted sampling scenario (favored by competitive accretion), the single massive cluster (bottom) has an overall larger gravitational potential well and consequently, the most massive star in the system will be a more massive star than three cluster system above containing an equal total mass. Conversely, in the stochastic sampling scenario (favored by core accretion), the sampling of the IMF is random, and therefore, since both the three small clusters and one large cluster have the same total mass, the most massive star will be the same in both systems.

\subsection{Competitive Accretion}

In the competitive accretion model (e.g.; Bonnell et al., 2003, 2007; Bate, 2012; Wang et al., 2010) for massive star formation, the global cluster potential is crucial for the formation of massive stars. Many fragments form in the turbulent initial conditions, some of which form low-mass stars, and others which are at preferential locations in the center of the gravitational potential well and continue to accrete as material funnels down into the potential (see Figure 3). The competitive accretion model requires the large-scale funneling of material into the central cluster potential. While the overall cluster-forming clump need not be globally collapsing, it at least needs to be bound to enable this process. The overall star forming process is dynamic occurs over several clump free-fall times.

In this model, the formation of a massive star (or stars) is always accompanied by the formation of a stellar cluster surrounding it. Therefore, we would expect strong and early mass segregation, and moreover, the formation of a massive star in isolation should not be possible. In fact, we would expect that the most massive stars can only form in the most massive clusters (because they have the largest gravitational potential well) and there should be a strong correlation between the maximum star mass in a cluster and the overall mass of the cluster. In other words, we would expect the sampling of the Initial Mass Function (IMF) to be sorted, massive clusters sample the high mass end of the IMF and lower mass clusters do not, rather than stochastic, every star forming region samples the IMF completely randomly, meaning that a massive star could form in isolation (see 


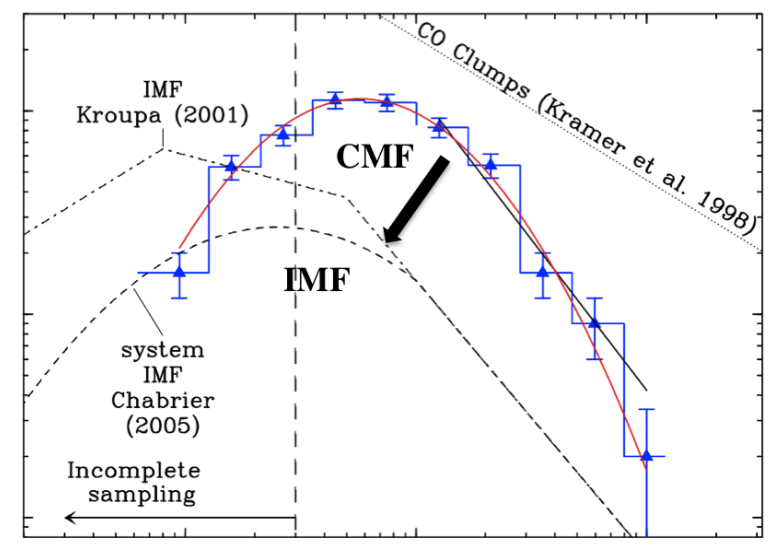

Figure 5: Observations show a close mapping of the CMF onto the IMF, modulo a factor of about three in efficiency. Shown here is the measured CMF in the Aquila Rift with Herschel (Andre et al., 2010) compared with the IMF. The mapping of the CMF onto the IMF is called into question based on biases in identifying cores (which are amorphous overdensities rather than discrete objects like stars) and the fact that many "cores" are seen to break up into smaller fragments at high resolution (e.g. Galván-Madrid et al., 2010) and are therefore likely to form more than a single, bound stellar system. Figure adapted from André et al. (2010).

Figure 4). These predictions can be tested by observing the correlation between the most massive star formed in a cluster $\left(\mathrm{M}_{* \max }\right)$ with the overall cluster mass $\left(\mathrm{M}_{\mathrm{cl}}\right)$. An extreme break in this correlation would be a massive star formed in isolation $\left(\mathrm{M}_{* \max }=\mathrm{M}_{\mathrm{cl}}\right)$, though less extreme breaks in the correlation would also be informative.

\section{Observational Tests of Massive Star Formation Theories}

I review here a few of the observational tests performed which may help to distinguish between competing theories of massive star formation, but begin with the simple conclusion here. Both theories aptly explain the known observables of massive star forming regions and clusters, such as the IMF, multiplicity fractions, and cluster properties (spatial and velocity distributions). However, of the new observables tested to distinguish between theories, no observational test or combination thereof are widely accepted to have proven or disproven either theory of massive star formation. Observations are ongoing and are consistently putting tighter constraints on both theories. It is important to note as well that as simulations improve to include more realistic physics, the gap between the competing theories lessens (e.g. Kruijssen, 2013).

\subsection{Does the Core Mass Function Map Directly onto the Initial Mass Function?}

Observational studies suggest that the Core Mass Function (CMF) is well-matched to the IMF, modulo a factor of about three, often attributed to the star-forming efficiency (e.g.; André et al., 2010, see Figure 5). While this is predicted by the core accretion theory, it is not disallowed by competitive accretion. Additionally, the physical meaning of observationally identified is questionable. Since a core is not a discrete, countable, physical object like a star, but rather an amorphous overdensity of gas and dust, its properties are inextricably tied with the nature of our observations 


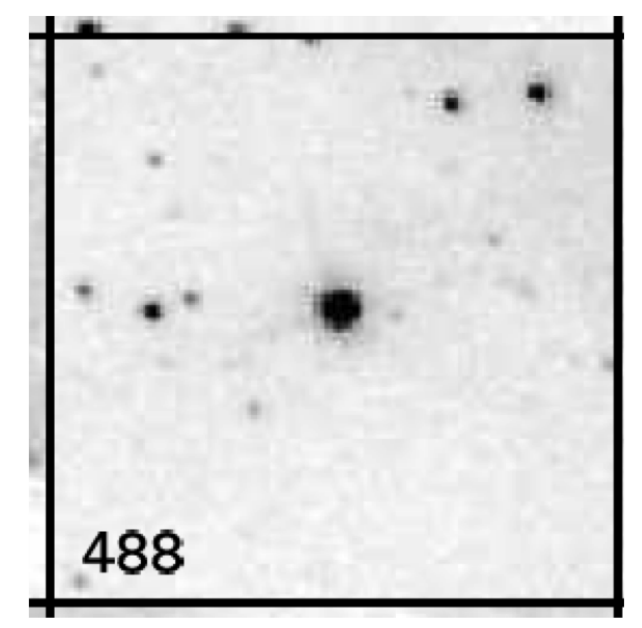

Figure 6: A candidate massive star formed in isolation from Bressert et al. (2012). This star is a $36 \mathrm{M}_{\odot} \mathrm{O}$ star located in 30 Doradus with no nearby cluster or evidence of ejection. Bressert et al. (2012) identify 15 such candidates and Oey et al. (2013); Lamb et al. (2010); de Wit et al. (2005) identify more. While the presence of such isolated systems is of potentially great importance, there remains some skepticism. Some claim that the candidate massive stars formed in isolation are consistent with simply being massive star runaways, rather than having formed in isolation (Oh et al., 2013; de Wit et al., 2005).

toward it and the tools used to extract it (e.g.; Pineda et al., 2009). In order for the mapping of the CMF onto the IMF to be meaningful, it must be demonstrated that the measured cores will each fragment to form a single stellar system (Offner et al., 2013). The most massive cores may be resolved into smaller cores (e.g.; Galván-Madrid et al., 2010) on closer inspection, which confuses a comparison of the observed CMF with the IMF.

\subsection{Sorted vs. Stochastic Sampling of the IMF}

As shown in Figure 4, sorted sampling is predicted in the model of competitive accretion, since the global cluster potential is the most important aspect affecting the mass of the most massive star. Alternatively, in the core accretion model, the IMF should be sampled stochastically. I present here two observations testing the mode of IMF sampling: 1) The correlation of the most massive star in a cluster with the mass of the cluster $\left(\mathrm{M}_{* \max }\right.$ vs. $\left.\mathrm{M}_{\mathrm{cl}}\right)$, and 2) the possible existence of massive stars formed in isolation $\left(\mathrm{M}_{* \max }=\mathrm{M}_{\mathrm{cl}}\right)$.

Observation 1: Some observations (e.g.; Weidner et al., 2013, 2010) show overwhelmingly strong evidence for sorted sampling. Weidner et al. (2013) show that the distribution of cluster $\mathbf{M}_{* \max }$ values is inconsistent with sampling randomly from the IMF with $99.9 \%$ confidence. However, it has been pointed out (e.g.; Lamb et al., 2010; Oey et al., 2013; Elmegreen, 2006) that observations are inherently insensitive to detecting small clusters with massive stars, as the massive star feedback would quickly evaporate any surrounding gas, making the detection of such a cluster more difficult. Therefore, there is strong evidence for a deviation from stochastic IMF sampling, but that effect may be perpetrated by observational biases.

Observation 2: There are a growing number of candidate massive stars thought to have formed in isolation (see Figure 6 and Oey et al., 2013; Lamb et al., 2010; Bressert et al., 2012; de Wit et al., 2005). While many tests have been performed to try to identify massive stars without 


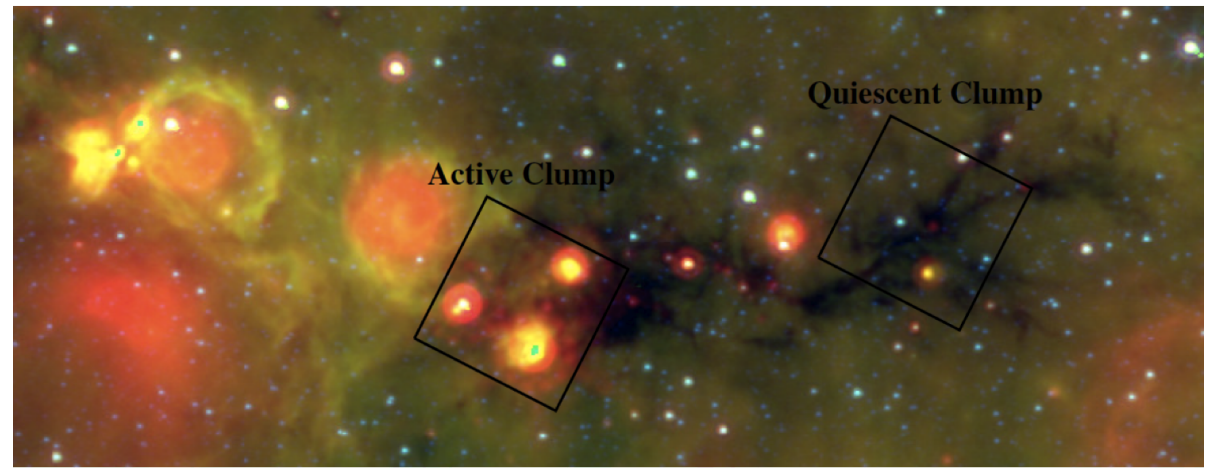

Figure 7: Massive star-forming IRDC G32.02+0.06 showing a range of evolutionary stages, from evolved $\mathrm{H}$ II regions at left to young, ultra-compact H II regions surrounded by warm, dense gas in the middle, to quiescent, cold, dense gas at the right. Image from Battersby et al. (2014). MIPSGAL $24 \mu \mathrm{m}$ shown in red (Carey et al., 2009), GLIMPSE 8.0 and $4.5 \mu \mathrm{m}$ shown in green and blue, respectively (Churchwell et al., 2009).

a signature of having been ejected from a nearby cluster, it is nearly impossible to prove that a single star formed in isolation. There are any number of possible scenarios in which a star may appear to be isolated, but was actually ejected from a cluster (e.g.; Oh et al., 2013; de Wit et al., 2005). Therefore, while no singular massive star candidate is sufficient to prove they can form in isolation, a significant number of candidates that exceeds our expectations for the number of ejected massive stars would be convincing evidence.

\subsection{Other Tests}

The observational tests presented in this section are by no means a complete census of work that has been done to discriminate between competing theories of massive star formation. Many other tests have been devised and are in progress. For example, ongoing studies using ALMA will shed light on the nature of disks around massive stars. Both theories predict $\sim 1000$ AU disks around massive stars, but in competitive accretion, massive stars will have more encounters that will destroy or truncate disks at regular intervals, meaning that some fraction will lack disks (e.g.; Moeckel \& Bally, 2006; Scally \& Clarke, 2001). The upper mass cutoff for stars (Elmegreen, 2000; Figer, 2005) is of great interest, as well as searching for variation in the IMF (e.g.; Conroy et al., 2013; Hopkins, 2013; Hennebelle, 2012). Finally, there is ongoing work studying the dynamical state of cluster-forming gas clumps (e.g. Kauffmann et al., 2013; Battersby et al., 2010).

Below, I review some work that has been done towards understanding the initial conditions for and the early evolutionary stages of massive star formation. Presently, simulations of massive star formation begin with different initial conditions, which are not always well motivated by observations. One way to improve our understanding of massive star formation is through a characterization of the early physical conditions and evolutionary stages for massive star formation observationally. Such observations not only serve as improved initial conditions for modeling massive star formation, but the physical conditions in early evolutionary stages are a fundamental observable and place significant constraints on models. 


\section{Probing Initial Conditions: The Sites of Massive Star Formation}

\subsection{Infrared Dark Clouds and Pre-Cluster Clumps}

Much like their low-mass counterparts, massive stars are thought to form in cold, dense molecular clouds; however, these natal clumps are necessarily of higher mass if they are to form massive stars. The discovery of Infrared Dark Clouds (IRDCs, see Figure 7) as dark extinction features against the bright mid-infrared Galactic background with the Midcourse Space Experiment (MSX) and the Infrared Space Observatory satellites (Egan et al., 1998; Perault et al., 1996; Omont et al., 2003) provided candidate massive star birthplaces. We will call these massive, dense gas clumps from which massive stars and clusters form Pre-Cluster Clumps (PCCs). By definition, IRDCs are cold and dense enough to obscure the Galactic mid-IR background at $8 \mu \mathrm{m}$, typically with temperatures $\mathrm{T}<25 \mathrm{~K}$ (Carey et al., 1998; Pillai et al., 2006) and densities $\mathrm{n}_{H}>10^{4} \mathrm{~cm}^{-3}$ (Egan et al., 1998; Carey et al., 1998; Rathborne et al., 2006). The most massive of these are about $10^{3}$ - $10^{4} \mathrm{M}_{\odot}$ (Rathborne et al., 2006; Battersby et al., 2010) and are substructures within larger Giant Molecular Clouds (GMCs).

In the past decade, IRDCs have emerged as likely sites for the birthplaces of massive stars, and many have been shown to host the earliest phases of massive star formation (e.g. Rathborne et al., 2006; Battersby et al., 2010; Ragan et al., 2006; Peretto \& Fuller, 2009). While the most massive IRDCs are likely PCC candidates, the majority of IRDCs (Simon et al., 2006; Peretto \& Fuller, 2009; Kauffmann \& Pillai, 2010; Wilcock et al., 2012) fall short of this threshold and are not capable of forming massive stars. Additionally, the identification of IRDCs suffers from a selection bias. Their identification against the bright mid-IR Galactic background precludes their identification in the outer Galaxy, at high Galactic latitudes, and on the far side of the Galaxy. IRDCs capable of forming massive stars (of which there are many) provide an excellent starting place for studies of massive star and cluster formation, however, Galactic-scale analyses of this process require another way to identify PCCs.

As the earliest phases of massive star and cluster formation occur in heavily embedded structures, it is necessary to observe at long-wavelengths to probe deep within the clump and measure the structure and properties of the star-forming cores. Recent long-wavelength surveys of the Galactic Plane allow us to peer into candidate PCCs across the Galaxy. Galactic Plane surveys in the sub-mm to far-IR, in particular, provide an optically thin tracer of the dust emission from these cold, dense clouds. These surveys include the Bolocam Galactic Plane Survey at $1.1 \mathrm{~mm}$ (BGPS Aguirre et al., 2011; Rosolowsky et al., 2010; Ginsburg et al., 2013), the APEX Telescope Large Area Survey of the Galaxy at $870 \mu \mathrm{m}$ (ATLASGAL Schuller et al., 2009), and the Herschel Infrared Galactic Plane Survey from 70 to $500 \mu \mathrm{m}$ (Hi-GAL Molinari et al., 2010). These surveys have confirmed that the most massive IRDCs are, in fact, cold, dense gas clumps seen in dust emission in the sub-mm (Battersby et al., 2010; Parsons et al., 2009; Wilcock et al., 2012). These long-wavelength surveys allow us to identify PCCs throughout the Galaxy in a systematic way, independent of their location with respect to the mid-IR background.

\subsection{Physical Properties and Evolution}

Investigations toward PCCs have found a range of temperatures, average densities, and starforming activity (e.g. Dunham et al., 2011a,b; Battersby et al., 2011; Wienen et al., 2012; Peretto 


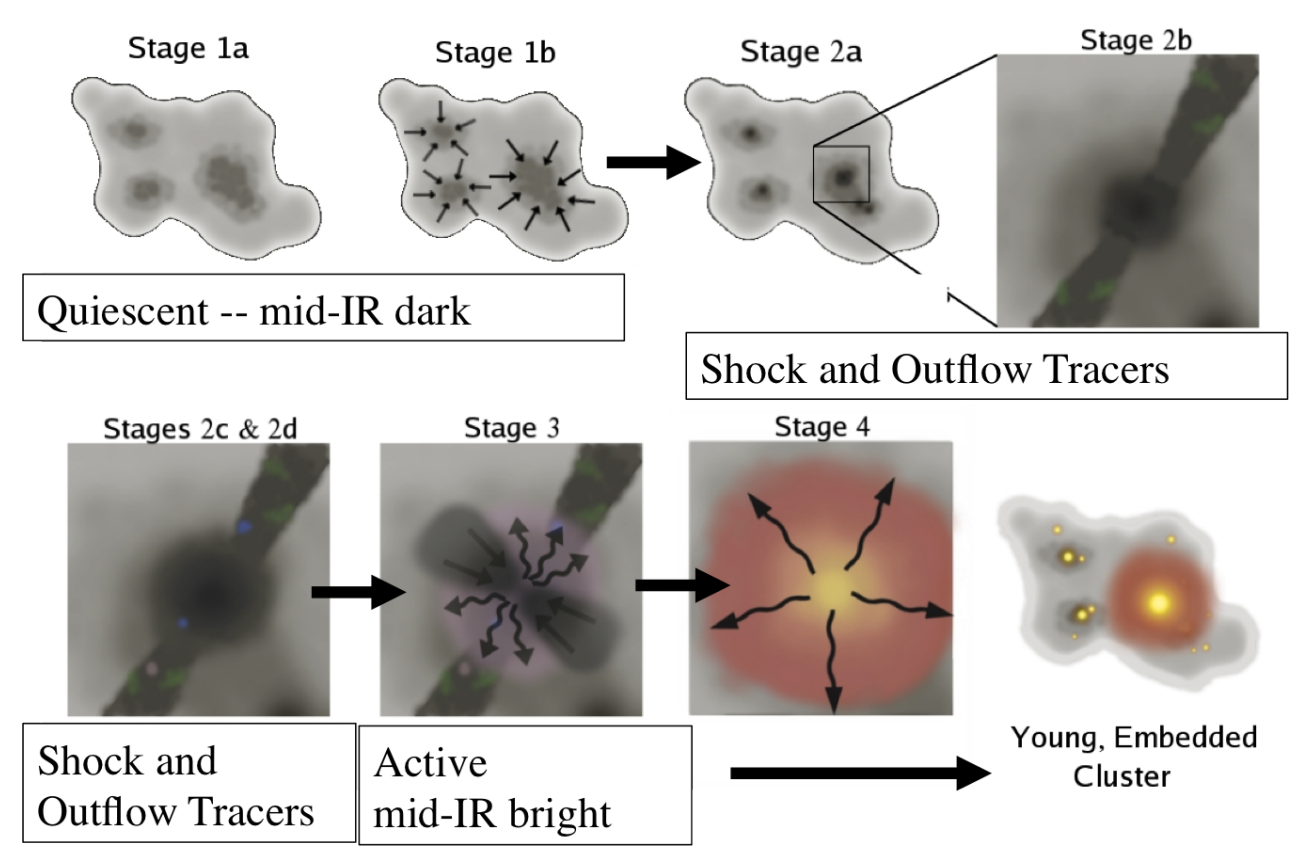

Figure 8: A cartoon depicting a proposed evolutionary sequence for massive star formation from Battersby et al. (2010). The sequence addresses the early signatures of massive star formation in cold, dense clumps of gas and dust identified in the sub-mm (through the thermal emission of cold, dense dust) or through their mid-IR extinction. The physical conditions progress from cold, dense, and quiescent, to showing signatures of shocks and outflows (seen through $6.7 \mathrm{GHz} \mathrm{CH} 3 \mathrm{OH}$ masers or Extended Green Objects; Cyganowski et al., 2008), to early embedded massive star formation seen through warm dust (such as emission at 8 or 24 $\mu \mathrm{m}$ ) or ultra-compact $\mathrm{H}$ II regions.

et al., 2010). While many of these dense gas clumps are cold and pre-star-forming, others are actively star-forming and have begun to heat up their natal molecular clump. Even IRDCs, thought to host the earliest stages of massive star formation, have been shown to exhibit a range of evolutionary states from pre- to actively star-forming (e.g. Chambers et al., 2009; Battersby et al., 2010).

Observations toward candidate PCCs have shown clumpy filamentary structure on $\sim 1 \mathrm{pc}$ scales punctuated by complexes of dense cores with masses in the range of $\sim 10-100 \mathrm{M}_{\odot}$ and separated by $\sim 0.1 \mathrm{pc}$ (though high-resolution observations suggest further fragmentation; GalvánMadrid et al., 2010; Brogan et al., 2009) with peak column densities of about 1-2 $\mathrm{g} \mathrm{cm}^{-2}$ (e.g. Zhang \& Wang, 2011; Rathborne et al., 2008; Devine et al., 2011; Wang et al., 2008; Battersby et al., 2014; Pillai et al., 2011; Fontani et al., 2012; Liu et al., 2012). The pre-star-forming cores and dense filaments have temperatures of about 10-20 K (Zhang et al., 2009; Rathborne et al., 2008; Pillai et al., 2011; Battersby et al., 2014) and the cores actively involved in star formation (but still with enough gas and dust to be detected in the dust continuum) show temperatures of $\mathrm{T}>35 \mathrm{~K}$ (Beuther et al., 2007; Zhang \& Wang, 2011; Battersby et al., 2014).

Battersby et al. (2010) proposed an evolutionary sequence (similar to Chambers et al., 2009; Purcell et al., 2009) for PCCs, beginning in the cold, dense, quiescent IRDC phase (see Figure 8), progressing into a phase showing early signatures of massive star formation (shocks and outflows as 


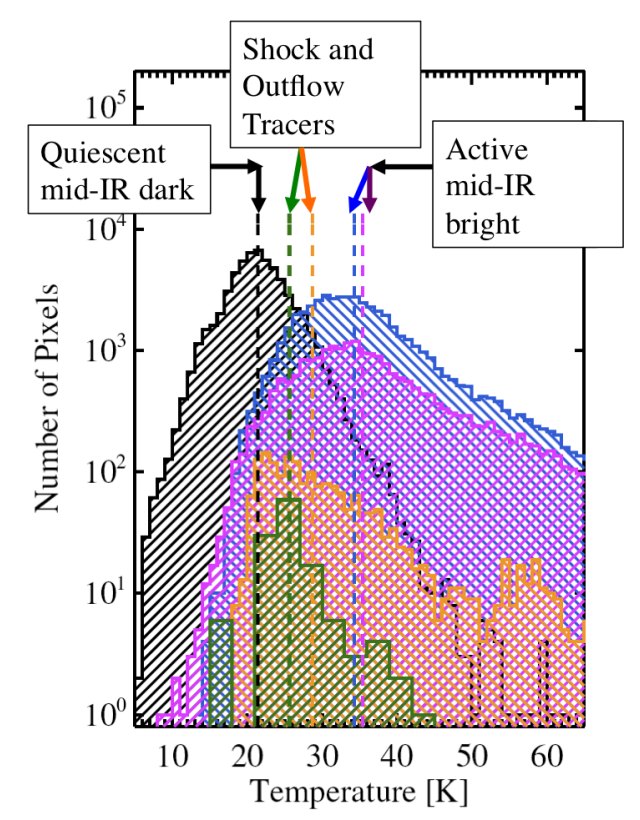

Figure 9: The progression of the proposed evolutionary sequence (see Figure 8) as a function of temperature from Battersby et al. (2011). If a clump monotonically increases in temperature with time, this progression from quiescent, mid-IR dark at the coldest temperatures to shock and outflow tracers to active and mid-IR dark at the warmest, indicates that we are seeing the evolution of clumps in our sample.

evidenced through Class II $\mathrm{CH}_{3} \mathrm{OH}$ maser emission and the appearance of Extended Green Objects Cyganowski et al., 2008), to young, embedded UCH II regions. The observation of monotonically increasing dust temperature with these phases (see Figure 9 and Battersby et al., 2011) supports the proposed evolutionary sequence.

While the general properties observed toward PCCs at high-resolution are well-constrained, the variance of these properties as a function of evolutionary stage or large-scale environment has yet to be clearly determined. A statistically significant correlation of physical properties with evolutionary stage will require large samples, similar to the analysis performed by Battersby et al. (2011), but at high resolution. The combination of the availability of large-scale Galactic Plane surveys and high-resolution facilities (e.g. SMA, CARMA, PdBI, ALMA, ATCA) makes such an analysis possible.

\section{Summary and Outlook}

The field of massive star formation has experienced prolific growth in the past decade and a half. As our ability to grasp the problem has grown, through increased computational power for numerical simulations and better wavelength coverage, resolution, sensitivity, and ability to perform large surveys, so has our interest. Numerical simulations are able to produce clusters of stars that fully sample the observed IMF, including massive stars, and closely match our observations of cluster-forming clumps. Competing theories suggest different mechanisms for the accretion of material onto the massive stars, both of which provide testable predictions. Observations are con- 
tinually putting strong constraints on the process of forming massive stars, but have yet to provide a definitive answer.

As simulations incorporate more realistic physics, include feedback from forming stars, and start with better and more consistent "initial conditions" the competing models will likely continue to converge. Improved observational prospects include the ability to observe large samples of massive star-forming clumps with Galactic plane surveys and probe massive star-forming cores at higher resolutions and better sensitivities than ever before. Our current observational capabilities should allow us to definitively test the predictions of competing models of massive star formation in the next decade; an exciting prospect.

Acknowledgements I thank the organizers of the Bash symposium for a productive and interesting meeting and for the opportunity to consider and expound on the status of our understanding of massive star and cluster formation.

\section{References}

Aguirre, J. E., Ginsburg, A. G., Dunham, M. K., et al. 2011, ApJS, 192, 4

André, P., Men'shchikov, A., Bontemps, S., et al. 2010, A\&A, 518, L102

Bate, M. R. 2012, MNRAS, 419, 3115

Battersby, C., Bally, J., Ginsburg, A., \& Longmore, S. 2014, ApJ, submitted

Battersby, C., Bally, J., Jackson, J. M., et al. 2010, ApJ, 721, 222

Battersby, C., Bally, J., Ginsburg, A., et al. 2011, A\&A, 535, A128

Bergin, E. A., \& Tafalla, M. 2007, ARA\&A, 45, 339

Beuther, H., Walsh, A. J., Thorwirth, S., et al. 2007, A\&A, 466, 989

Bonnell, I. A., Bate, M. R., Clarke, C. J., \& Pringle, J. E. 2001, MNRAS, 323, 785

Bonnell, I. A., Bate, M. R., \& Vine, S. G. 2003, MNRAS, 343, 413

Bonnell, I. A., Larson, R. B., \& Zinnecker, H. 2007, Protostars and Planets V, 149

Bressert, E., Bastian, N., Evans, C. J., et al. 2012, A\&A, 542, A49

Brogan, C. L., Hunter, T. R., Cyganowski, C. J., et al. 2009, ApJ, 707, 1

Carey, S. J., Clark, F. O., Egan, M. P., et al. 1998, ApJ, 508, 721

Carey, S. J., Noriega-Crespo, A., Mizuno, D. R., et al. 2009, PASP, 121, 76

Chambers, E. T., Jackson, J. M., Rathborne, J. M., \& Simon, R. 2009, ApJS, 181, 360

Churchwell, E., Babler, B. L., Meade, M. R., et al. 2009, PASP, 121, 213

Conroy, C., Dutton, A. A., Graves, G. J., Mendel, J. T., \& van Dokkum, P. G. 2013, ApJL, 776, L26

Cyganowski, C. J., Whitney, B. A., Holden, E., et al. 2008, AJ, 136, 2391 
de Wit, W. J., Testi, L., Palla, F., \& Zinnecker, H. 2005, A\&A, 437, 247

Devine, K. E., Chandler, C. J., Brogan, C., et al. 2011, ApJ, 733, 44

Dunham, M. K., Robitaille, T. P., Evans, II, N. J., et al. 2011a, ApJ, 731, 90

Dunham, M. K., Rosolowsky, E., Evans, II, N. J., Cyganowski, C., \& Urquhart, J. S. 2011b, ApJ, 741, 110

Egan, M. P., Shipman, R. F., Price, S. D., et al. 1998, ApJL, 494, L199

Elmegreen, B. G. 2000, ApJ, 530, 277

-. 2006, ApJ, 648, 572

Figer, D. F. 2005, Nature, 434, 192

Fontani, F., Caselli, P., Zhang, Q., et al. 2012, A\&A, 541, A32

Galván-Madrid, R., Zhang, Q., Keto, E., et al. 2010, ApJ, 725, 17

Ginsburg, A., Glenn, J., Rosolowsky, E., et al. 2013, ArXiv e-prints, arXiv:1305.6622

Hennebelle, P. 2012, A\&A, 545, A147

Hopkins, P. F. 2013, MNRAS, 433, 170

Kauffmann, J., \& Pillai, T. 2010, ApJL, 723, L7

Kauffmann, J., Pillai, T., \& Zhang, Q. 2013, ApJL, 765, L35

Kruijssen, J. M. D. 2013, ArXiv e-prints, arXiv:1304.4600

Krumholz, M. R., \& Bonnell, I. A. 2007, ArXiv e-prints, arXiv:0712.0828

Krumholz, M. R., Klein, R. I., \& McKee, C. F. 2007, ApJ, 656, 959

Krumholz, M. R., Klein, R. I., McKee, C. F., Offner, S. S. R., \& Cunningham, A. J. 2009, Science, 323, 754

Lada, C. J., \& Lada, E. A. 2003, ARA\&A, 41, 57

Lamb, J. B., Oey, M. S., Werk, J. K., \& Ingleby, L. D. 2010, ApJ, 725, 1886

Liu, H. B., Jiménez-Serra, I., Ho, P. T. P., et al. 2012, ApJ, 756, 10

McKee, C. F., \& Tan, J. C. 2003, ApJ, 585, 850

McLaughlin, D. E., \& Pudritz, R. E. 1996, ApJ, 469, 194

Moeckel, N., \& Bally, J. 2006, ApJ, 653, 437

Molinari, S., Swinyard, B., Bally, J., et al. 2010, A\&A, 518, L100

Oey, M. S., Lamb, J. B., Kushner, C. T., Pellegrini, E. W., \& Graus, A. S. 2013, ApJ, 768, 66

Offner, S. S. R., Clark, P. C., Hennebelle, P., et al. 2013, ArXiv e-prints, arXiv:1312.5326

Oh, S., Kroupa, P., \& Banerjee, S. 2013, ArXiv e-prints, arXiv:1311.2934 
Omont, A., Gilmore, G. F., Alard, C., et al. 2003, A\&A, 403, 975

Parsons, H., Thompson, M. A., \& Chrysostomou, A. 2009, MNRAS, 399, 1506

Perault, M., Omont, A., Simon, G., et al. 1996, A\&A, 315, L165

Peretto, N., \& Fuller, G. A. 2009, A\&A, 505, 405

Peretto, N., Fuller, G. A., Plume, R., et al. 2010, A\&A, 518, L98+

Pillai, T., Kauffmann, J., Wyrowski, F., et al. 2011, A\&A, 530, A118

Pillai, T., Wyrowski, F., Carey, S. J., \& Menten, K. M. 2006, A\&A, 450, 569

Pineda, J. E., Rosolowsky, E. W., \& Goodman, A. A. 2009, ApJL, 699, L134

Purcell, C. R., Longmore, S. N., Burton, M. G., et al. 2009, MNRAS, 394, 323

Ragan, S. E., Bergin, E. A., Plume, R., et al. 2006, ApJS, 166, 567

Rathborne, J. M., Jackson, J. M., \& Simon, R. 2006, ApJ, 641, 389

Rathborne, J. M., Jackson, J. M., Zhang, Q., \& Simon, R. 2008, ApJ, 689, 1141

Rosolowsky, E., Dunham, M. K., Ginsburg, A., et al. 2010, ApJS, 188, 123

Scally, A., \& Clarke, C. 2001, MNRAS, 325, 449

Schuller, F., Menten, K. M., Contreras, Y., et al. 2009, A\&A, 504, 415

Shu, F. H., Adams, F. C., \& Lizano, S. 1987, ARA\&A, 25, 23

Simon, R., Jackson, J. M., Rathborne, J. M., \& Chambers, E. T. 2006, ApJ, 639, 227

Wang, P., Li, Z.-Y., Abel, T., \& Nakamura, F. 2010, ApJ, 709, 27

Wang, Y., Zhang, Q., Pillai, T., Wyrowski, F., \& Wu, Y. 2008, ApJL, 672, L33

Weidner, C., Kroupa, P., \& Bonnell, I. A. D. 2010, MNRAS, 401, 275

Weidner, C., Kroupa, P., \& Pflamm-Altenburg, J. 2013, MNRAS, 434, 84

Wienen, M., Wyrowski, F., Schuller, F., et al. 2012, A\&A, 544, A146

Wilcock, L. A., Ward-Thompson, D., Kirk, J. M., et al. 2012, MNRAS, 422, 1071

Zhang, Q., \& Wang, K. 2011, ApJ, 733, 26

Zhang, Q., Wang, Y., Pillai, T., \& Rathborne, J. 2009, ApJ, 696, 268 